\title{
Cigarette Exposure, Dependence, and Craving Are Related to Insula Thickness in Young Adult Smokers
}

\author{
Angelica M Morales ${ }^{1,2,3}$, Dara Ghahremani ${ }^{2}$, Milky Kohno ${ }^{1,2,3}$, Gerhard S Hellemann ${ }^{2}$ and \\ Edythe D London*,2,3,4 \\ 'Neuroscience Interdepartmental Program, University of California, Los Angeles, Los Angeles, CA, USA; ${ }^{2}$ Department of Psychiatry and \\ Biobehavioral Sciences, University of California, Los Angeles, Los Angeles, CA, USA; ${ }^{3}$ Brain Research Institute, University of California, Los Angeles, \\ Los Angeles, CA, USA; ${ }^{4}$ Department of Molecular and Medical Pharmacology, University of California, Los Angeles, Los Angeles, CA, USA
}

\begin{abstract}
The age period spanning late adolescence to emergent adulthood is associated with the highest prevalence of cigarette smoking in the United States, and is also a time of continued brain development. Nonetheless, although prior research has shown group differences in brain structure associated with smoking status in adults, few studies have examined how smoking and associated behavioral states relate to brain structure in this age group. Neuroimaging and lesion studies have suggested that the insula, a cortical region that integrates heterogeneous signals about internal states and contributes to executive functions, plays an important role in cigarette smoking behavior. Using high-resolution structural magnetic resonance imaging, we therefore measured cortical thickness of the insula in 18 smokers and 24 nonsmokers between the ages of 16 and 21 years. There were no group differences in insula thickness, but cigarette exposure (packyears) was negatively associated with thickness in right insula. Cigarette dependence and the urge to smoke were negatively related to cortical thickness in the right ventral anterior insula. Although the results do not demonstrate causation, they do suggest that there are effects of cigarette exposure on brain structure in young smokers, with a relatively short smoking history. It is possible that changes in the brain due to prolonged exposure or to the progression of dependence lead to more extensive structural changes, manifested in the reported group differences between adult smokers and nonsmokers. Structural integrity of the insula may have implications for predicting long-term cigarette smoking and problems with other substance abuse in this population.
\end{abstract}

Neuropsychopharmacology (2014) 39, I8I6-I822; doi:I0.1038/npp.20।4.48; published online 26 March 2014

\section{INTRODUCTION}

Young people in the age range of late adolescence to emerging adulthood (18 to 25 years of age) have the highest prevalence of cigarette smoking in the United States, and $\sim 33 \%$ are considered smokers (USDHHS, 2012). Most smokers start smoking in their teenage years, with half of adolescent smokers transitioning into heavy smoking during emerging adulthood (White et al, 2009). During adolescence and emergent adulthood, the brain is still undergoing development (Sowell et al, 2003), and it has been hypothesized that tobacco use during this critical period produces neurobiological changes that promote tobacco dependence later in life (DeBry and Tiffany, 2008). Understanding the relationship between smoking behavior and gray-matter integrity in emergent adults, therefore, has the potential to help clarify the neurobiological mechanisms that underlie

*Correspondence: Dr ED London, Semel Institute of Neuroscience and Human Behavior, University of California, Los Angeles, 760 Westwood Plaza, Los Angeles, CA 90024-1759, USA, Tel: + 310825 0606, Fax: + 3108250812

E-mail: elondon@mednet.ucla.edu

Received I October 2013; revised 7 January 2014; accepted 21 February 2014; accepted article preview online 3 March 2014 the transition to tobacco dependence in adulthood and to aid in the development of treatment strategies tailored for this large subpopulation of smokers in the United States (Stone et al, 2012).

Converging lines of evidence suggest that the insular cortex plays a critical role in the maintenance of tobacco dependence (Naqvi and Bechara, 2010). Within the human cerebral cortex, the insula has the highest density of nicotinic acetylcholine receptors, as indicated by positron emission tomography using $2-\left[{ }^{18} \mathrm{~F}\right] \mathrm{F}-\mathrm{A}-85380$ (Picard et al, 2013), a tracer for nicotinic acetylcholine receptors containing $\beta 2$ subunits (Kimes et al, 2003). Damage to the insular cortex, but not to other parts of the brain, disrupts smoking behavior in stroke patients and animals (Naqvi et al, 2007; Scott and Hiroi, 2011). Conversely, many studies have shown that smoking-related cues elicit increases in glucose metabolism and blood oxygenated level-dependent (BOLD) signal in a network of brain regions including the insula (Brody et al, 2002; Garavan, 2010). Moreover, self-reported cigarette craving is related to change in glucose metabolism of the anterior insular cortex in response to visual cues (Brody et al, 2002), and to cerebral blood flow in the insula elicited by overnight abstinence from smoking (Wang et al, 2007). It therefore appears that the insula, particularly the anterior portion, plays an integral role in generating the 
conscious urge to smoke. Despite progress in understanding how the function of the insula affects smoking behavior, less is known about the importance of individual differences in the structural integrity of the insula.

Structural magnetic resonance imaging (sMRI) has not been used to examine gray-matter integrity in young smokers despite evidence that exposure to nicotine produces cell damage and loss in the brains of adolescent animals (Slotkin, 2002). The majority of studies in adult smokers find that individuals who smoke have lower regional gray-matter volume or density than nonsmokers in various brain regions (Brody et al, 2004; Morales et al, 2012) including the insular cortex (Gallinat et al, 2006; Zhang et al, 2011b). A single study, however, found greater gray-matter density in the left insula in smokers than nonsmokers (Zhang et al, 2011a). Inconsistencies in the literature may be attributable to the structural and functional heterogeneity of the insula. The insula has been divided into three subregions on the basis of cytoarchitectonic information in macaques (Mesulam and Mufson, 1982a), and on the basis of resting-state functional connectivity, task-evoked activation, and structural connectivity (Deen et al, 2011; Kelly et al, 2012) in humans. Previous studies have indicated a role of the dorsal anterior insula in cognition and executive control, the ventral anterior insula in emotion, and the posterior insula in the reception of primary interoceptive and exteroceptive information (Chang et al, 2013; Craig, 2002).

In the current study, therefore, we measured cortical thickness in the whole insula and in three insula subregions, and we hypothesized that smokers in emergent adulthood would have thinner insular cortex than nonsmokers in the same age group. Although insula lesions disrupt smoking behavior, duration of nicotine dependence is negatively correlated with insula volume in adult polysubstance abusers (Tanabe et al, 2013); suggesting that there is a complicated relationship between insula structure and function. Based on these studies, we predicted that cigarette exposure and dependence would be negatively correlated with insula thickness. As the ventral and dorsal anterior insula are structurally and functionally connected to the orbitofrontal cortex, anterior cingulate (ACC), and dorsolateral prefrontal cortex (Deen et al, 2011; Mesulam and Mufson, 1982b), regions commonly implicated in craving and in the ability to control the urge to smoke (Hayashi et al, 2013; Kober et al, 2010; Li et al, 2013), we hypothesized that thickness of the anterior insula would be negatively correlated with the urge to smoke.

\section{MATERIALS AND METHODS}

\section{Participants}

As described in a previous report (Galvan et al, 2011), participants were recruited through online and print advertisements. After receiving a detailed explanation of the study (approved by the UCLA Institutional Review Board), participants $\geqslant 18$ years of age gave written informed consent. Those under the age of 18 years gave assent, and their parents provided written informed consent. A total of 24 English-speaking, right-handed nonsmokers and 18 daily smokers completed study procedures. No participant reported a medical or neurological disorder that would affect brain function or structure. The Structured Clinical Interview for DSM-IV (First et al, 1995) was used to exclude participants meeting diagnosis for any Axis I psychiatric disorder, including current drug abuse or dependence (except nicotine for the smokers). Abstinence from substance use (except nicotine for smokers) was confirmed by urine drug screening on test days.

Participants were classified as nonsmokers if they selfreported smoking of $<5$ cigarettes in their lifetime, had carbon monoxide (CO) concentrations of $<5$ p.p.m. in expired air (Smokelyzer, Bedfont Scientific, Kent, UK), and had urinary cotinine values below the threshold of determination (NicAlert test strips, Nymox Pharmaceutical, Hasbrouck Heights, NJ). Smokers endorsed daily cigarette smoking, had CO concentrations $\geqslant 6$ p.p.m. in expired air, and/or urinary cotinine $\geqslant 200 \mathrm{ng} / \mathrm{ml}$. Pack-year smoking history, accounting for periods of abstinence and fluctuations in average number of cigarettes smoked per day, was calculated to determine lifetime exposure to cigarettes (pack years $=$ packs smoked per day $\times$ years as a smoker). Severity of cigarette dependence was assessed with the Cigarette Dependence Scale (CDS-12; (Etter et al, 2003)). Participants were only asked to refrain from smoking during the $30 \mathrm{~min}$ immediately preceding the scan (average duration of abstinence before scan: $258 \pm 248 \mathrm{~min}$ ).

\section{Scan Acquisition}

A high-resolution magnetization-prepared rapid-acquisition gradient echo (MPRAGE) was acquired on a 3T Siemens Trio MRI scanner for measurement of cortical thickness (TR, 2.3; TE, 2.1; FOV, 256; matrix, $192 \times 192$; sagittal plane; slice thickness, $1 \mathrm{~mm}$; 160 slices).

\section{sMRI Preprocessing}

The FreeSurfer image analysis suite (version 5.0) was used for semiautomatic measurement of cortical thickness from MPRAGE images. The methods, fully described elsewhere (Dale et al, 1999; Fischl and Dale, 2000; Fischl et al, 1999), are briefly described here. The intensity of the images was normalized to remove bias fields and a watershed algorithm was applied to remove non-brain tissue (Segonne et al, 2004). White matter was segmented from the rest of the brain, and errors in white-matter topology were corrected automatically (Fischl et al, 2001). A tessellation was formed along the boundary between gray and white matter. The tessellation on the white-matter surface was grown outward toward the intensity gradient separating the gray matter from the cerebrospinal fluid (pial surface). White-matter and pial surfaces were visually inspected for accuracy and manual corrections were made in cases where the white matter was not accurately classified (mostly in temporal lobes) and in cases where the pial surface included dura or skull. Finally, the cortical surfaces were mapped onto a spherical coordinate system and registered to an average template by maximizing the correspondence between surface convexity measures.

Whole insula thickness was determined using the Desikan-Killiany Atlas (Desikan et al, 2006). The insula was parcellated into three subdivisions based on boundaries determined via functional parcellation studies (Deen et al, 
2011). Dorsal anterior, ventral anterior, and posterior insula labels were created on an average template (Figure 1) and then mapped back to individual subjects in order to measure average cortical thickness within each of the three subdivisions from each participant's data. The posterior and anterior insula were divided along the left/right plane of the anterior commissure (MNI coordinate: $\mathrm{Y}=4$ ). Dorsal and ventral anterior insula were delineated in the coronal plane based on the convexity of the gyri in anterior insula (MNI coordinates: $\mathrm{Z}=-4$ ).

\section{Statistical Analysis}

Thickness values were extracted from gray-matter regions of interest (ROIs) and were imported into SPSS v20. Group comparisons were conducted using analysis of covariance (ANCOVA) with thickness as the dependent variable, group as a between-subjects factor with two levels (smoker/ nonsmoker), and age, sex, alcoholic drinks per week, and frequency of marijuana use as continuous covariates. Correlations were conducted to test the relationships between thickness values, pack-years, and CDS-12 scores. Multiple-comparison correction was performed by controlling for the rate of false discoveries (FDRs; $5 \% \alpha$-level) (Benjamini and Hochberg, 1995). The insular cortex is thought to play an integral role in generating the conscious urge to smoke: therefore, exploratory post hoc analyses correlated responses from the CDS question 'After a few hours without smoking I feel an irresistible urge to smoke' with thickness in ROIs that correlated with the total CDS score. Alcoholic drinks per week and frequency of marijuana use were not correlated with pack-years, CDS scores, or thickness; therefore, they were not included as covariates in the partial correlations. To determine the neuroantomical specificity of correlations between thickness and pack-years or CDS scores, secondary statistical analyses were conducted vertex-wise across the whole brain. Maps were smoothed using a Gaussian kernel of 10-mm full-width half maximum and Monte Carlo simulations were performed to identify significant clusters (cluster forming threshold $p<0.01$; cluster-wise significance threshold $p<0.05$ ).

\section{RESULTS}

\section{Participant Characteristics}

No differences were detected between smokers and nonsmokers in sex distribution $\left(\chi^{2} \quad(1,42)=0.008\right.$, $p=0.93)$, age $(t(40)=-1.02, p=0.32)$, Weschler Adult Intelligence Scale (WAIS) combined score $(t(40)=0.06$ $p=0.95)$, years of education $(t(40)=-0.47, p=0.64)$, or ethnicity $\left(\chi^{2}(1,42)=6.21, p=0.18\right)$. Young smokers and nonsmokers differed on alcoholic drinks per week $(t(40)=-2.90, p=0.009$, unequal variance) and on marijuana use in the past 30 days $(t(40)=-2.33, p=0.032$, unequal variance). On average, young adult smokers started smoking at 15.1 years of age $(\mathrm{SD}=2.4)$, smoked 6.7 $(\mathrm{SD}=2.3)$ cigarettes per day, had a $0.9(\mathrm{SD}=0.7)$ packyear smoking history, and a cigarette dependence score of $35.0(\mathrm{SD}=7.3$; Table 1$)$.

\section{Cortical Thickness Results}

No differences in cortical thickness were detected between nonsmokers and smokers in bilateral insula thickness (Figure 2a) or in the thickness of bilateral dorsal anterior,

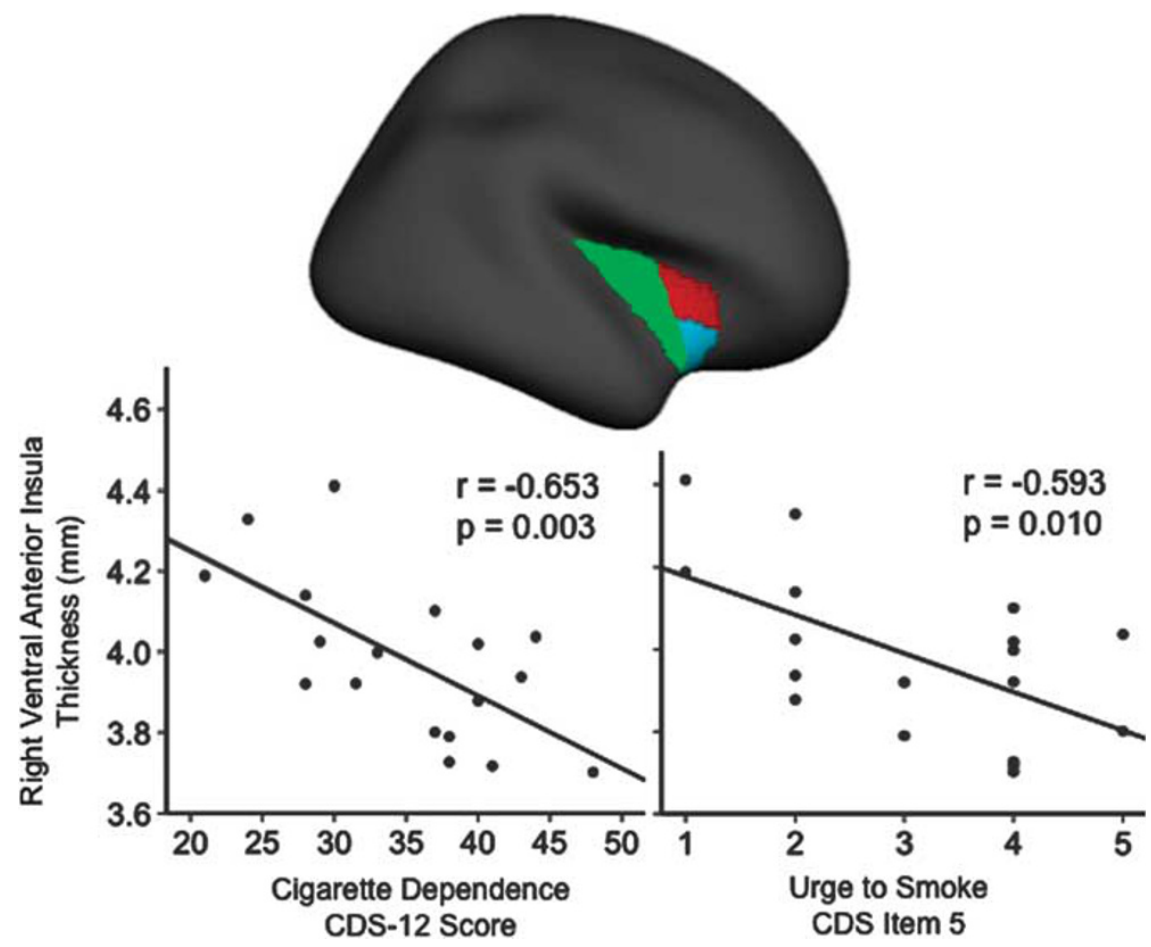

Figure I Relationships between cigarette dependence, craving, and insula thickness. The insula was parcellated into three subregions: dorsal anterior (red), ventral anterior (blue), and posterior insula (green). Correlations show that right ventral anterior insula thickness is negatively correlated with total score on the Cigarette Dependence Scale (CDS-12) and the urge to smoke after a few hours without smoking. 
ventral anterior, or posterior insula ( $p$ 's $>0.4$ ). In data from young adult smokers, pack-year smoking history was negatively correlated with the thickness of the right insula

Table I Participant Characteristics ${ }^{a}$

\begin{tabular}{|c|c|c|}
\hline & $\begin{array}{c}\text { Nonsmokers } \\
\quad(n=24)\end{array}$ & $\begin{array}{c}\text { Smokers } \\
(n=18)\end{array}$ \\
\hline $\operatorname{Sex}(M / F)$ & $|3 / 1|$ & $10 / 8$ \\
\hline Age (years) & $19.5(1.4)$ & $19.9(1.3)$ \\
\hline WAIS combined score & $93.3(10.6)$ & $93.1(6.4)$ \\
\hline Education (years) & $13.4(1.4)$ & |3.6 (1.2) \\
\hline \multicolumn{3}{|l|}{ Ethnicity } \\
\hline White Caucasian & 4 & 6 \\
\hline African American & 5 & 1 \\
\hline Hispanic & 2 & 5 \\
\hline Asian & 9 & 4 \\
\hline Other & 4 & 2 \\
\hline Marijuana (days used in past 30 days) ${ }^{b}$ & $0.08(0.3)$ & $0.9(1.6)$ \\
\hline Alcohol (drinks/week) $)^{b}$ & $0.8(1.3)$ & $4.0(4.6)$ \\
\hline \multicolumn{3}{|l|}{ Cigarette smoking } \\
\hline Age of first use (years) & & |5.| (2.4) \\
\hline Cigarettes per day & & $6.6(2.4)$ \\
\hline Pack-years & & $0.9(0.7)$ \\
\hline Cigarette dependence score & & $35.0(7.3)$ \\
\hline
\end{tabular}

aMean (SD).

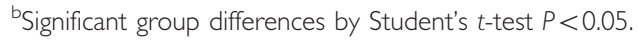

( $r=-0.639, p=0.004$; FDR threshold for significance: $p<0.00625$, Figure $2 \mathrm{~b}$ ), but no correlation was detected on the left $(r=-0.059, p=0.817$, Figure 2b). Pack-year smoking history was also negatively correlated with right dorsal anterior $(r=-0.581, p=0.011)$ and right posterior insula $(r=-0.497, p=0.036)$ thickness; however, these associations did not reach statistical significance after controlling for the rate of false discoveries. CDS scores did not correlate with whole insula thickness (right hemisphere: $r=-0.301, p=0.224$; left hemisphere: $r=-0.035, p=0.892$ ), but were negatively correlated with right ventral anterior insula thickness $(r=-0.653, p=0.003$; FDR threshold for significance: $p<0.003125$, Figure 1$)$, with a similar trend observed in the right dorsal anterior insula $(r=-0.507$, $p=0.032)$. Similarly, the urge to smoke was correlated with right ventral anterior insula thickness $(r=-0.593, p=0.01$; uncorrected, Figure 1). No other correlations were detected and whole-brain voxel-wise analyses did not yield any significant results.

\section{DISCUSSION}

This study provides evidence that cigarette exposure, dependence, and craving are related to thickness of the insula in emergent adults with relatively little smoking experience. Although brain structure has been studied extensively in adult smokers, studying the neural correlates of smoking behavior in emerging adulthood is of utmost importance, as it may help us understand why smoking
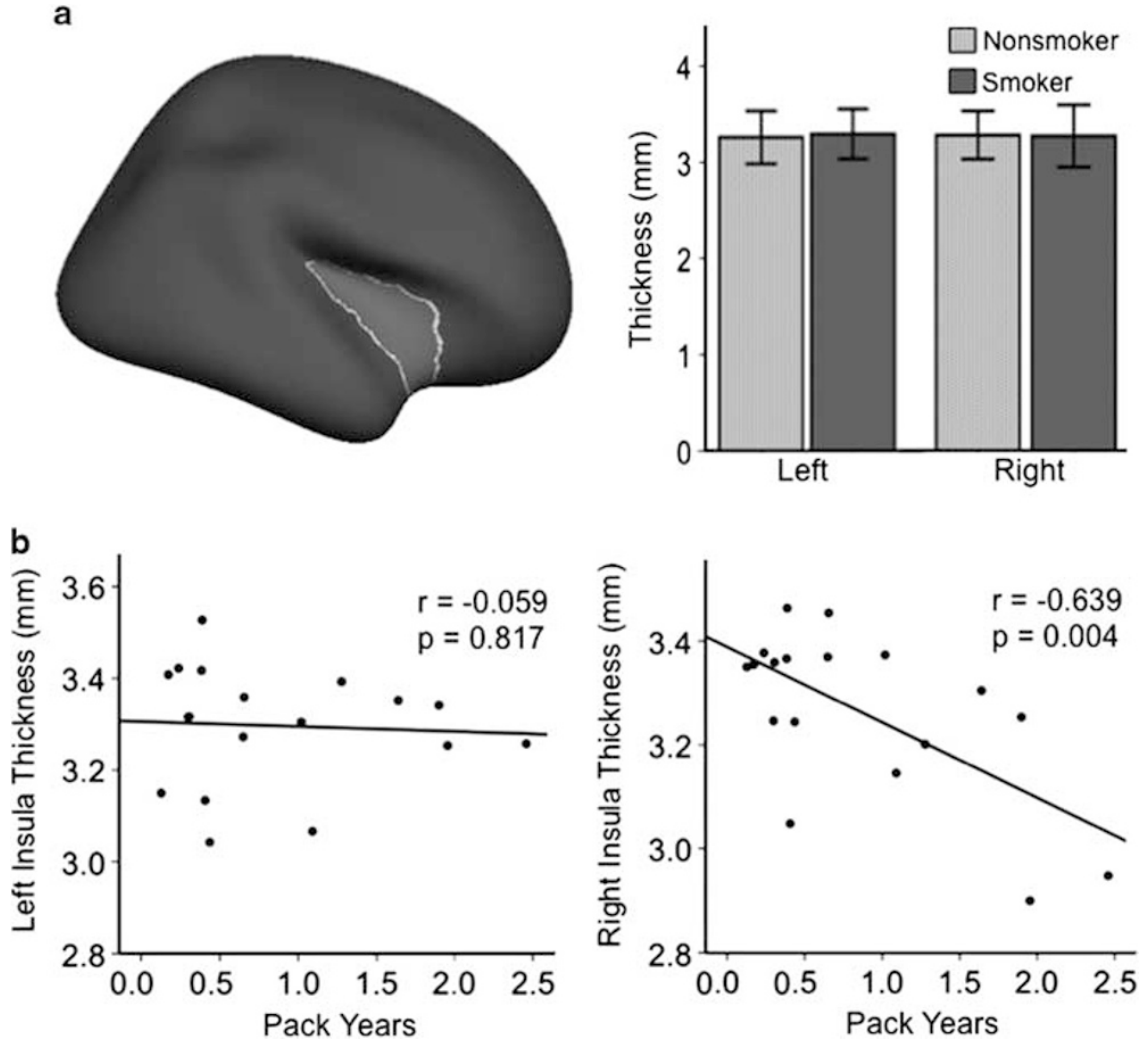

Figure 2 Group differences in whole insula thickness and correlations of insula thickness with cigarette exposure. In the anatomically defined right and left insula regions of interest (left hemisphere depicted in (a)), there were no statistically significant group differences in average thickness (error bars: mean \pm 2 SD). Correlations show that pack-year smoking history was correlated with right but no correlation was detected with left insula thickness (b). 
during this developmental epoch has such a profound impact on lifelong smoking behavior (Sussman, 2002). Unlike studies in adult smokers (Brody et al, 2002; Morales et al, 2012), this examination of young participants did not reveal group differences in cortical thickness between smokers and nonsmokers, suggesting that differences seen in adults develop as a result of prolonged cigarette smoking or that they arise later in the developmental trajectory because of other factors.

Negative correlations between cortical thickness and cigarette exposure may be attributable to the neurotoxic effects of nicotine or to other constituents of tobacco smoke. Participants in this study began smoking in adolescence (mean age of initiation: 15.1 years old), and studies in animals indicate that exposure to nicotine during adolescence reduces the number of cells in the cerebral cortex by $5-10 \%$ as indexed by DNA content; however, DNA content was measured in fairly gross anatomical regions precluding determination of anatomical specificity and possibly masking the true magnitude of the effect (Slotkin, 2002). Effects of continued exposure may explain reports of lower gray-matter density in insula in adult smokers than nonsmokers (Gallinat et al, 2006; Zhang et al, 2011a). Although one study reports greater insula graymatter density in adult smokers than nonsmokers, left insula density was correlated with symptoms of alexithymia, but not with cigarette exposure or dependence (Zhang et al, 2011a), suggesting that other factors comorbid with smoking may contribute to the discrepant results. Differences in the methods used to index gray-matter integrity could also contribute to the heterogeneity in the literature.

This study focused on the insular cortex because of its central role in the maintenance of tobacco dependence; however, studies of adult smokers suggest that cigarette use is associated with gray-matter abnormalities throughout the brain. These studies have shown a dose-dependent effect of tobacco exposure on gray-matter integrity in prefrontal cortex (Zhang et al, 2011a), temporal cortex, and cerebellum (Gallinat et al, 2006); however, such effects were not observed in whole-brain vertex-wise analyses conducted in this sample. These discrepancies may be attributable to the low levels of exposure in this sample ( 0.9 pack-years on average). Notably, a study of adult smokers found that individuals with high levels of exposure (18.6 pack-years on average), but not those with lower levels of exposure (7.2 pack-years on average), had lower gray matter density in the prefrontal cortex than nonsmokers (Zhang et al, 2011a). Furthermore, correlations between exposure, dependence, and prefrontal gray-matter density were only present in the high-exposure group (Zhang et al, 2011a).

The CDS assesses an individual's subjective experience of symptoms such as craving, compulsion to use, levels of stress when unable to smoke, and difficulty quitting or controlling intake (Etter et al, 2003). Overall experience of these subjective feeling states and the severity of the urge to smoke were negatively correlated with the thickness of the right ventral anterior insula, a brain region that integrates primary interoceptive information with emotional information and that is believed to generate conscious awareness of feeling states (Craig, 2002; Gray and Critchley, 2007). Although this is the first report to link cigarette dependence and craving to insula thickness, studies of adult smokers have found that craving is negatively correlated with restingstate functional connectivity between the right anterior insula and the ventromedial prefrontal cortex (Sutherland et al, 2013). More work is necessary to determine whether individual differences in gray-matter thickness influence the circuit-level interactions of the insula with other brain regions implicated in craving.

Future studies should directly examine neurobiological differences between the right and left insula in smokers. Although differences between right and left insula were not explicitly tested in this study, results indicate that cortical thickness of the right insula is related to cigarette exposure, dependence and craving, but no correlations between smoking behavior and left insula thickness were detected, suggesting that the right insula may be particularly relevant to smoking behavior. Although lesions to both the right and left insula disrupt smoking behavior, a greater proportion of those with right insula lesions experience a disruption in smoking behavior (Naqvi et al, 2007). Another study found that rsFC connectivity between the right (but not left) anterior insula and ventromedial prefrontal cortex was related to tobacco craving and alexithymia (Sutherland et al, 2013). Given the importance of the anterior cingulate in smoking behavior (Azizian et al, 2010; Sharma and Brody, 2009; Sutherland et al, 2012), it is also of interest that the right ventral anterior insula shows greater rsFC with anterior cingulate cortex than left ventral anterior insula (Cauda et al, 2011). Despite this evidence, however, the left insular cortex is also implicated in cigarette dependence (Gallinat et al, 2006; Moran et al, 2012) and studies specifically designed to clarify the differential roles of right and left insula in cigarette smoking behavior are needed.

Neuroimaging and animal studies suggest that cigarette smoking is linked to other aspects of brain structure and function in adolescence and emergent adulthood. Adolescent smokers exhibit higher fractional anisotropy (FA) in the brain than nonsmokers, and FA is positively correlated with cigarette exposure (Jacobsen et al, 2007). These differences in white matter microstructure persist into adulthood (Hudkins et al, 2012) and may be related to the effects of nicotine on the expression of myelin genes (Cao et al, 2013) or on oligodendrocytes precursor cells (Bartzokis, 2007). Studies in adolescent animals also show that exposure to nicotine results in suppressed neural activity and persistent desensitization of cholinergic responses (Slotkin, 2008). Furthermore, prior studies by our research group using functional magnetic resonance imaging to study a subset of the participants in the present study have shown that the Heaviness of Smoking Index is related to brain activation during response inhibition and risky decision making in emergent adults (Galvan et al, 2011; Galvan et al, 2013), suggesting that alterations in brain function related to smoking may have a meaningful impact on behavior. Together, these studies begin to reveal a holistic picture of the effects of smoking on the developing brain, and future work to integrate these findings may lead to a better understanding of the neural mechanisms that support addiction.

This work is not without limitations. The cross-sectional study design makes it impossible to dissociate causal effects of cigarette exposure and dependence from biological susceptibility factors that promote drug use. Our sample was relatively small, precluding our ability to test how other 
factors, such as sex (Brown et al, 2012) and genetic vulnerability for tobacco addiction (Hong et al, 2010), may influence the relationship between smoking and brain measures. Although nonsmoking and smoking groups differed on alcoholic drinks per week and frequency of marijuana use, these variables were included in statistical models used to test for group comparisons. Alcoholic drinks per week and frequency of marijuana use were not correlated with packyears or cigarette dependence scores and correlations between clinical features of cigarette use and brain structure remained significant with these variables included in the statistical model. Furthermore, levels of alcohol and marijuana use were low in all groups and no participants met DSM-IV criteria for substance abuse or dependence.

In emergent adult smokers, anterior insula thickness is negatively correlated with cigarette exposure, dependence, and craving. We found no group differences in insula thickness, suggesting that interventions that result in early smoking cessation may prevent the development of neurobiological differences commonly observed between adult smokers and nonsmokers. Prospective studies are needed to determine whether abnormalities in insula thickness during emergent adulthood serve as a neurobiological susceptibility factor for cigarette dependence in adulthood. This study extends previous studies that implicate the insula in addiction among adult smokers, as these results may reflect the initial effects of cigarette smoking on insula thickness.

\section{FUNDING AND DISCLOSURE}

The authors declare no conflict of interest. The research described in this article was funded, in part, by a grant from Philip Morris USA to Dr London under UCLA contract (number 20063287). Additional funding was provided by an endowment from the Thomas P and Katherine K Pike Chair in Addiction Studies and a gift from the Marjorie M Greene Trust. AM Morales and M Kohno were supported by institutional training grant T32 DA 024635 and by F31 DA0331-17 and F31 DA033120-02, respectively. None of the sponsors were involved with the design, collection, analysis or interpretation of data, writing the manuscript, or the decision to submit the manuscript for publications. ED London, the principal investigator, takes responsibility for the integrity of the data and the accuracy of the data analysis. All of the authors had full access to all the data in the study.

\section{REFERENCES}

Azizian A, Nestor LJ, Payer D, Monterosso JR, Brody AL, London ED (2010). Smoking reduces conflict-related anterior cingulate activity in abstinent cigarette smokers performing a Stroop task. Neuropsychopharmacology 35: 775-782.

Bartzokis G (2007). Acetylcholinesterase inhibitors may improve myelin integrity. Biol Psychiatry 62: 294-301.

Benjamini Y, Hochberg Y (1995). Controlling the false discovery rate - a practical and powerful approach to multiple testing. $J$ R Stat Soc Ser B Method 57: 289-300.

Brody AL, Mandelkern MA, Jarvik ME, Lee GS, Smith EC, Huang JC et al (2004). Differences between smokers and nonsmokers in regional gray matter volumes and densities. Biol Psychiatry 55: 77-84.
Brody AL, Mandelkern MA, London ED, Childress AR, Lee GS, Bota RG et al (2002). Brain metabolic changes during cigarette craving. Arch Gen Psychiatry 59: 1162-1172.

Brown AK, Mandelkern MA, Farahi J, Robertson C, Ghahremani DG, Sumerel B et al (2012). Sex differences in striatal dopamine D2/D3 receptor availability in smokers and non-smokers. Int $J$ Neuropsychopharmacol 15: 989-994.

Cao J, Wang J, Dwyer JB, Gautier NM, Wang S, Leslie FM et al (2013). Gestational nicotine exposure modifies myelin gene expression in the brains of adolescent rats with sex differences. Transl Psychiatry 3: e247.

Cauda F, D’Agata F, Sacco K, Duca S, Geminiani G, Vercelli A (2011). Functional connectivity of the insula in the resting brain. Neuroimage 55: 8-23.

Chang LJ, Yarkoni T, Khaw MW, Sanfey AG (2013). Decoding the role of the insula in human cognition: functional parcellation and large-scale reverse inference. Cereb Cortex 23: 739-749.

Craig AD (2002). How do you feel? Interoception: the sense of the physiological condition of the body. Nat Rev Neurosci 3: 655-666.

Dale AM, Fischl B, Sereno MI (1999). Cortical surface-based analysis. I. Segmentation and surface reconstruction. Neuroimage 9: 179-194.

DeBry SC, Tiffany ST (2008). Tobacco-induced neurotoxicity of adolescent cognitive development (TINACD): a proposed model for the development of impulsivity in nicotine dependence. Nicotine Tob Res 10: 11-25.

Deen B, Pitskel NB, Pelphrey KA (2011). Three systems of insular functional connectivity identified with cluster analysis. Cereb Cortex 21: 1498-1506.

Desikan RS, Segonne F, Fischl B, Quinn BT, Dickerson BC, Blacker $\mathrm{D}$ et al (2006). An automated labeling system for subdividing the human cerebral cortex on MRI scans into gyral based regions of interest. Neuroimage 31: 968-980.

Etter JF, Le Houezec J, Perneger TV (2003). A self-administered questionnaire to measure dependence on cigarettes: the cigarette dependence scale. Neuropsychopharmacology 28: 359-370.

First MB, Spitzer RL, Gibbon M, Williams JB (1995). The Structured Clinical Interview for DSM-IV Axis I Disorders (SCID-IP). American Psychiatric Press: Washington, DC.

Fischl B, Dale AM (2000). Measuring the thickness of the human cerebral cortex from magnetic resonance images. Proc Natl Acad Sci USA 97: 11050-11055.

Fischl B, Liu A, Dale AM (2001). Automated manifold surgery: constructing geometrically accurate and topologically correct models of the human cerebral cortex. IEEE Trans Med Imaging 20: $70-80$.

Fischl B, Sereno MI, Dale AM (1999). Cortical surface-based analysis. II: Inflation, flattening, and a surface-based coordinate system. Neuroimage 9: 195-207.

Gallinat J, Meisenzahl E, Jacobsen LK, Kalus P, Bierbrauer J, Kienast $\mathrm{T}$ et al (2006). Smoking and structural brain deficits: a volumetric MR investigation. Eur J Neurosci 24: 1744-1750.

Galvan A, Poldrack RA, Baker CM, McGlennen KM, London ED (2011). Neural correlates of response inhibition and cigarette smoking in late adolescence. Neuropsychopharmacology 36: 970-978.

Galvan A, Schonberg T, Mumford J, Kohno M, Poldrack RA, London ED (2013). Greater risk sensitivity of dorsolateral prefrontal cortex in young smokers than in nonsmokers. Psychopharmacology (Berl) 229: 345-355.

Garavan H (2010). Insula and drug cravings. Brain Struct Funct 214: 593-601.

Gray MA, Critchley HD (2007). Interoceptive basis to craving. Neuron 54: 183-186.

Hayashi T, Ko JH, Strafella AP, Dagher A (2013). Dorsolateral prefrontal and orbitofrontal cortex interactions during self- 
control of cigarette craving. Proc Natl Acad Sci USA 110: $4422-4427$.

Hong LE, Hodgkinson CA, Yang Y, Sampath H, Ross TJ, Buchholz B et al (2010). A genetically modulated, intrinsic cingulate circuit supports human nicotine addiction. Proc Natl Acad Sci USA 107: 13509-13514.

Hudkins M, O’Neill J, Tobias MC, Bartzokis G, London ED (2012). Cigarette smoking and white matter microstructure. Psychopharmacology (Berl) 221: 285-295.

Jacobsen LK, Picciotto MR, Heath CJ, Frost SJ, Tsou KA, Dwan RA et al (2007). Prenatal and adolescent exposure to tobacco smoke modulates the development of white matter microstructure. J Neurosci 27: 13491-13498.

Kelly C, Toro R, Di Martino A, Cox CL, Bellec P, Castellanos FX et al (2012). A convergent functional architecture of the insula emerges across imaging modalities. Neuroimage 61: 1129-1142.

Kimes AS, Horti AG, London ED, Chefer SI, Contoreggi C, Ernst M et al (2003). 2-[18F]F-A-85380: PET imaging of brain nicotinic acetylcholine receptors and whole body distribution in humans. FASEB J 17: 1331-1333.

Kober H, Mende-Siedlecki P, Kross EF, Weber J, Mischel W, Hart CL et al (2010). Prefrontal-striatal pathway underlies cognitive regulation of craving. Proc Natl Acad Sci USA 107: 14811-14816.

Li X, Hartwell KJ, Borckardt J, Prisciandaro JJ, Saladin ME, Morgan PS et al (2013). Volitional reduction of anterior cingulate cortex activity produces decreased cue craving in smoking cessation: a preliminary real-time fMRI study. Addict Biol 18: 739-748.

Mesulam MM, Mufson EJ (1982a). Insula of the old world monkey. I. Architectonics in the insulo-orbito-temporal component of the paralimbic brain. J Comp Neurol 212: 1-22.

Mesulam MM, Mufson EJ (1982b). Insula of the old world monkey. III: Efferent cortical output and comments on function. J Comp Neurol 212: 38-52.

Morales AM, Lee B, Hellemann G, O’Neill J, London ED (2012). Gray-matter volume in methamphetamine dependence: cigarette smoking and changes with abstinence from methamphetamine. Drug Alcohol Depend 125: 230-238.

Moran LV, Sampath H, Stein EA, Hong LE (2012). Insular and anterior cingulate circuits in smokers with schizophrenia. Schizophr Res 142: 223-229.

Naqvi NH, Bechara A (2010). The insula and drug addiction: an interoceptive view of pleasure, urges, and decision-making. Brain Struct Funct 214: 435-450.

Naqvi NH, Rudrauf D, Damasio H, Bechara A (2007). Damage to the insula disrupts addiction to cigarette smoking. Science 315: 531-534.

Picard F, Sadaghiani S, Leroy C, Courvoisier DS, Maroy R, Bottlaender M (2013). High density of nicotinic receptors in the cingulo-insular network. Neuroimage 79: 42-51.
Scott D, Hiroi N (2011). Deconstructing craving: dissociable cortical control of cue reactivity in nicotine addiction. Biol Psychiatry 69: 1052-1059.

Segonne F, Dale AM, Busa E, Glessner M, Salat D, Hahn HK et al (2004). A hybrid approach to the skull stripping problem in MRI. Neuroimage 22: 1060-1075.

Sharma A, Brody AL (2009). In vivo brain imaging of human exposure to nicotine and tobacco. Handb Exp Pharmacol 192: 145-171.

Slotkin TA (2002). Nicotine and the adolescent brain: insights from an animal model. Neurotoxicol Teratol 24: 369-384.

Slotkin TA (2008). If nicotine is a developmental neurotoxicant in animal studies, dare we recommend nicotine replacement therapy in pregnant women and adolescents? Neurotoxicol Teratol 30: 1-19.

Sowell ER, Peterson BS, Thompson PM, Welcome SE, Henkenius AL, Toga AW (2003). Mapping cortical change across the human life span. Nat Neurosci 6: 309-315.

Stone AL, Becker LG, Huber AM, Catalano RF (2012). Review of risk and protective factors of substance use and problem use in emerging adulthood. Addict Behav 37: 747-775.

Sussman S (2002). Smoking cessation among persons in recovery. Subst Use Misuse 37: 1275-1298.

Sutherland MT, Carroll AJ, Salmeron BJ, Ross TJ, Stein EA (2013). Insula's functional connectivity with ventromedial prefrontal cortex mediates the impact of trait alexithymia on state tobacco craving. Psychopharmacology (Berl) 228: 143-155.

Sutherland MT, McHugh MJ, Pariyadath V, Stein EA (2012). Resting state functional connectivity in addiction: lessons learned and a road ahead. Neuroimage 62: 2281-2295.

Tanabe J, York P, Krmpotich T, Miller D, Dalwani M, Sakai JT et al (2013). Insula and orbitofrontal cortical morphology in substance dependence is modulated by sex. AJNR Am J Neuroradiol 34: $1150-1156$.

USDHHS (2012). Preventing tobacco use among youth and young adults: a report of the surgeon general. In U.S. Department of Health and Human Services, Centers for Disease Control and Prevention, National Center for Chronic Disease Prevention and Health Promotion, Office on Smoking and Health, Services $\mathrm{UDoHaH}(\mathrm{ed})$.

Wang Z, Faith M, Patterson F, Tang K, Kerrin K, Wileyto EP et al (2007). Neural substrates of abstinence-induced cigarette cravings in chronic smokers. J Neurosci 27: 14035-14040.

White HR, Bray BC, Fleming CB, Catalano RF (2009). Transitions into and out of light and intermittent smoking during emerging adulthood. Nicotine Tob Res 11: 211-219.

Zhang X, Salmeron BJ, Ross TJ, Geng X, Yang Y, Stein EA (2011a). Factors underlying prefrontal and insula structural alterations in smokers. Neuroimage 54: 42-48.

Zhang X, Salmeron BJ, Ross TJ, Gu H, Geng X, Yang Y et al (2011b). Anatomical differences and network characteristics underlying smoking cue reactivity. Neuroimage 54: 131-141. 